\title{
Reply to "Perioperative Alcohol Withdrawal Syndrome: Care Based on Empathy plus Prescriptions Based on Physiology"
}

\author{
Alexander Lavinius Ungur ${ }^{a}$ Tim Neumann $^{b, c}$ Friedrich Borchers ${ }^{a}$ \\ Claudia Spies $^{\text {a }}$ \\ ${ }^{a}$ Department of Anesthesiology and Intensive Care Medicine, Campus Charité Mitte and Campus Virchow-Klinikum,

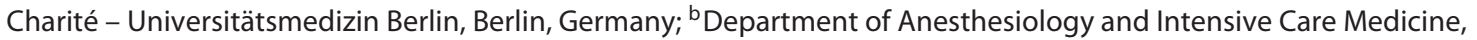 \\ Campus Benjamin Franklin, Charité - Universitätsmedizin Berlin, Berlin, Germany; ${ }^{\circ}$ QUEST Center for Transforming \\ Biomedical Research, Berlin Institute of Health, Berlin, Germany
}

\section{Dear Editor,}

We want to thank Dr. Braillon for his insightful comments [1]. We also want to thank the editor because this offers us the opportunity to discuss and deepen certain clinical and ethical aspects of the perioperative management of alcohol withdrawal syndrome.

Firstly, the importance of care was stressed by Dr. Braillon in order to prevent delirium, especially the importance of non-pharmacological measures in a setting with organizational problems. Indeed, non-pharmacological measures of delirium therapy are emphasized in current guidelines and reviews, as we stated in our second-last paragraph on page 161 . Of course, limited staff and training often leads to significant deficits in nonpharmacologic measures of delirium prevention and therapy, especially in (stressful) ICU settings. Several recent articles have been published on this specific topic and the importance of this aspect is obvious. We would have discussed non-pharmacological measures of delirium prevention to a much larger extent if the limit of the editorial frame of this article had allowed us to.

Secondly, in his comment Dr. Braillon also underlined the role of other preexisting concomitant medical problems like infection, pain, and especially smoking. Here, he suggested prescribing nicotine replacement therapy and supplemental sedatives, neuroleptics, and physical restraints. The role of smoking is emphasized as a known comorbidity of alcohol use disorders. In our ICUs, we do use transdermal nicotine replacement therapy on a pa- tient's demand and if there is no evidence of coronary heart disease. Otherwise, we prefer other pharmacologic agents to treat nicotine withdrawal, if necessary. In our standard operating procedures, first-line agents for treatment of vegetative arousal caused by nicotine withdrawal are, besides non-pharmacologic measures, alpha-2 agonists.

It is not clear why ventilated critically ill smokers necessitate (!) physical restraints. In 2010, Lucidarme et al. [2] themselves wrote that "restraints were left to the attending physician's judgment, and thus, were likely to be somewhat random." One has to keep in mind that physical restraints might have severe side effects. The risk/etiology of delirium in smokers is complex and has been questioned by Hsieh et al. [3]. Routine nicotine replacement therapy cannot be recommended for prevention of delirium or to reduce hospital or ICU mortality in critically ill smokers according to a 2017 systematic review, where high-quality data was not available [4].

Thirdly, prevention of thiamine deficiency and concurrent morbidity was discussed and commented on. We advise the use of thiamine in several parts of our article for prophylaxis as well as for therapy of alcohol withdrawal patients. Prophylactic administration is advised for all malnourished alcohol use disorder patients. If signs of delirium occur, we suggest high dose administration of parenteral thiamine every $8 \mathrm{~h}$, as advised by US, British, and also German guidelines (see the third paragraph on page 165). Thus, we totally agree with your statement 
even if we did not discuss the pathophysiology and the aspect of magnesium co-medication.

Lastly, Dr. Braillon questioned the following statement: "Writing down alcohol-related diagnoses in the medical records requires the patient's presumed consent after shared decision-making." He is concerned about censoring medical records' most critical data, which might worsen clinical outcomes. Our comment to this point is, as ICU physicians, we always need to find a compromise between a "paternalistic" and a "liberal" way to care for our patients, especially if their ability to contribute to shared decision making is impaired by deliriumlike conditions. Certainly, during recent years we have seen a paradigm shift towards patient autonomy. In Germany, all medical records are considered the patient's property. The European Union released the General Data Protection Regulation of 2018, where the subject's full control over all saved personal data is clarified. This principle is to be seen independently of the confidentiality agreement of medical data. If writing down certain diagnoses in the medical reports could be disadvantageous (i.e., due to stigmatization by other healthcare actors, legal prosecution organs, insurance companies, etc. - they all could, under certain circumstances, get access to medical reports), we have to weigh up the pros and cons before making them visible in the records. This weighing up is, in our eyes, not a censoring if made by the autonomous acting patient. This "shared decision making" might also strengthen the patient-doctor relation and could have a therapeutical benefit, as one may meet the spirit of motivational interviewing by exchanging information, expressing empathy, developing discrepancy, rolling with resistance, and supporting self-efficacy. This could have a value not only for individuals with alcohol use disorders.

\section{Conflict of Interest Statement}

The authors have no specific conflicts of interest resulting from financial involvement.

\section{References}

1 Braillon A. Perioperative alcohol withdrawal syndrome: care based on empathy plus prescriptions based on physiology. Visc Med. 2021. doi: $10.1159 / 000513299$.

2 Lucidarme O, Seguin A, Daubin C, Ramakers $\mathrm{M}$, Terzi N, Beck P, et al. Nicotine withdrawal and agitation in ventilated critically ill patients. Crit Care. 2010;14(2):R58.
3 Hsieh SJ, Shum M, Lee AN, Hasselmark F, Gong MN. Cigarette smoking as a risk factor for delirium in hospitalized and intensive care unit patients. A systematic review. Ann Am Thor Surg. 2013;10(5):496-503.

$4 \mathrm{Ng} \mathrm{KT}$, Gillies M, Griffith DM. Effect of nicotine replacement therapy on mortality, delirium, and duration of therapy in critically ill smokers: a systematic review and meta-analysis. Anaesth Intensive Care. 2017 Sep;45(5): 556-61. 